\title{
Comments on: Farkas' lemma: three decades of generalizations for mathematical optimization
}

\author{
Chong Li • K. F. Ng
}

Published online: 1 February 2014

(C) Sociedad de Estadística e Investigación Operativa 2014

The celebrated Farkas lemma originated in Farkas (1902) provides us an attractively simple and extremely useful characterization for a linear inequality to be a consequence of a linear inequality system on the Euclidean space $\mathbb{R}^{n}$. This lemma has been extensively studied and extended in many directions, including conic systems (linear, sublinear, convex), infinite/semi-infinite convex inequality systems and some classes of nonconvex systems such as the difference of convex (DC) systems and composite convex systems, and the rich results are spread in the literature. A brief survey on earlier extensions was made by Jeyakumar (2008); the authors, Dinh and Jeyakumar, of the present survey and Jeyakumar (2008) have done a good job in drawing a global picture to show us how the celebrated Farkas' lemma is developed and extended in the last three decades emerging into a major field in the modern convex analysis and the variational analysis theory. This survey contains seven main parts. The first six parts are concerned with the generalized Farkas' lemma under some mild constraint qualifications for systems from simple cone linear inequality/equality systems in Banach spaces to composite convex inequality systems and some more complicated special nonconvex inequality systems; while the last part is with the sequential forms of the generalized Farkas' lemma for these systems without any qualification. One main

This comment refers to the invited paper available at doi:10.1007/s11750-014-0319-y.

C. Li $(\bowtie)$

Department of Mathematics, Zhejiang University, Hangzhou 310027,

People's Republic of China

e-mail: cli@zju.edu.cn

K. F. Ng

Department of Mathematics (\& IMS), Chinese University of Hong Kong,

Hong Kong, People's Republic of China

e-mail: kfng@math.cuhk.edu.hk 
feature is on the Robust Farkas' lemma under data uncertainty, while another important feature is on the effective use of the following epigraph-type qualifications for inequality systems:

$$
\bigcup_{y^{*} \in K^{+}} \operatorname{epi}\left(f+y^{*} \circ g+\delta_{C}\right)^{*} \text { is } w^{*} \text {-closed, }
$$

and

$$
\bigcup_{\lambda \in \mathbb{R}_{+}^{(T)}} \operatorname{epi}\left(f+\delta_{C}+\sum_{t \in T} \lambda_{t} f_{t}(x)\right)^{*} \text { is } w^{*} \text {-closed; }
$$

where $C \subseteq X$ is closed convex, $f, f_{t}: X \rightarrow \mathbb{R}$ are proper convex and lower semicontinuous for each $t \in T$, and $g: X \rightarrow Y^{\bullet}$ is $K$-convex and $K$-epi-closed. The qualifications (1) and (2) are utilized to establish the following generalized Farkas' lemmas in Dinh et al. (2013) and Fang et al. (2010), respectively; see also Theorems 4.1 and 4.4 of this survey.

Theorem 1 Assume qualification (1). Then, for any $\beta \in \mathbb{R}$, the following statements are equivalent:

(i) $x \in C, g(x) \in-K \Longrightarrow f(x) \geq \beta$.

(ii) $\exists y^{*} \in K^{+}$such that $f+y^{*} \circ g \geq \beta$ on $C$.

Theorem 2 Assume qualification (2). Then, for any $\beta \in \mathbb{R}$, the following statements are equivalent:

(i) $x \in C, f_{t}(x) \leq 0, \forall t \in T \Longrightarrow f(x) \geq \beta$.

(ii) $\exists\left(\lambda_{t}\right)_{t \in T} \in \mathbb{R}_{+}^{(T)}$ such that $f(x)+\sum_{t \in T} \lambda_{t} f_{t}(x) \geq \beta$ on $C$.

In light of these results, it is worthy for us to say a few words in the remainder of this discussion on sufficient conditions ensuring the epigraph-type qualification (1) or (2).

Consider a nonempty convex set $D$ of $X$. Recall that the core of $D$ and the intrinsic core of $D$, denoted by $\operatorname{cor} D$ and icr $D$, are defined by

$$
\operatorname{cor} D:=\{d \in D:(\forall x \in X)(\exists \varepsilon>0)(\forall \lambda \in[-\varepsilon, \varepsilon]) d+\lambda x \in D\}
$$

and

$$
\operatorname{icr} D:=\{d \in D:(\forall x \in \operatorname{aff} D)(\exists \varepsilon>0)(\forall \lambda \in[-\varepsilon, \varepsilon]) d+\lambda x \in D\},
$$

respectively. Recall also that the strong quasi-relative interior of $D$ is denoted by sqri $D$ and defined to be the set of all $x \in D$ such that cone $(D-x)$ is a closed subspace.

Jeyakumar et al. (2004) presented the following generalized Slater's (interior-point) constraint qualifications:

(a) $0 \in \operatorname{icr}(g(C)+S)$ and aff $(g(C)+S)$ is a closed subspace;

(b) $0 \in \operatorname{sqri}(g(C)+S)$;

(c) $0 \in \operatorname{core}(g(C)+S)$;

(d) $0 \in g(C)+\operatorname{int} S$. 
As shown in Jeyakumar et al. (2004), the following implications hold in the case when $X$ and $Y$ are Banach spaces:

$$
(\mathrm{d}) \Longrightarrow(\mathrm{c}) \Longrightarrow(\mathrm{b}) \Longleftrightarrow(\mathrm{a}) \text {. }
$$

The following proposition regards a possible implication that the generalized Slater's constraint qualification (a) implies (1).

Proposition 1 Let $X$ and $Y$ be Banach spaces, and let $g: X \rightarrow Y$ be continuous. Suppose that the constraint qualification (a) holds. Then qualification (1) holds if $\operatorname{int}(\operatorname{dom} f) \cap C \cap g^{-1}(K) \neq \varnothing$.

Indeed, under the assumptions of the above proposition, Jeyakumar et al. (2004) established the closed cone constraint qualification (CCCQ):

$$
\bigcup_{y^{*} \in K^{+}} \operatorname{epi}(y \circ g)^{*}+\operatorname{epi} \delta_{C}^{*} \text { is } w^{*} \text {-closed. }
$$

Thus, by (Fang et al. 2010, Lemma 2.1(ii)), one can conclude that qualification (1) holds because $f$ is continuous at any point in int $(\operatorname{dom} f)$.

To state some useful sufficient conditions for qualification (2), we recall some Slater type conditions for the system $\left\{f_{t}: t \in T\right\}$, introduced in Li et al. (2013) for the case when $X:=\mathbb{R}^{n}$. Let $D$ and $C$ be two convex subsets of $X$. Let $T\left(x_{0}\right):=\{t \in T$ : $\left.f_{t}\left(x_{0}\right)=0\right\}$ for $x_{0} \in D$. The system $\left\{f_{t}: t \in T\right\}$ is said to satisfy

(a) the Slater condition ${ }^{1}$ on $D$ if there exists a point $x_{0} \in D$ such that $\sup _{t \in T} f_{t}\left(x_{0}\right)<0$

(b) the weak Slater condition on $D$ if there exists a point $x_{0} \in D$ such that

$$
\sup _{t \in T \backslash T\left(x_{0}\right)} f_{t}\left(x_{0}\right)<0, \quad f_{t} \text { is affine for each } t \in T\left(x_{0}\right)
$$

and $T\left(x_{0}\right)$ is finite;

(c) the $C$-quasi Slater condition on $D$ if there exists a point $x_{0} \in D$ such that (3) and the following closure condition hold:

$$
\text { cone }\left\{\partial f_{t}\left(x_{0}\right): t \in T\left(x_{0}\right)\right\}+\left(\operatorname{span}\left(C-x_{0}\right)\right)^{\perp} \text { is } w^{*} \text {-closed. }
$$

As explained in Li et al. (2013), the Slater condition on $D$ implies the weak Slater condition on $D$, which implies the $C$-quasi Slater condition on $D$. Then we have the following proposition which provides some sufficient conditions for qualification (2) to hold.

Proposition 2 Let $X=\mathbb{R}^{n}$ and set $A:=\left\{x \in C: f_{t}(x) \leq 0 \forall t \in T\right\}$. Suppose that

\footnotetext{
1 This condition is also referred as the "strong Slater condition" in some literature (e.g., Goberna and López 1998).
} 
(i) $T$ is a compact metric space, and the function $t \mapsto f_{t}(x)$ is upper semicontinuous on $T$ for each $x \in$ aff $C$;

(ii) the system $\left\{f_{t}: t \in T\right\}$ satisfies the Slater condition on $C$ or the $C$-quasi Slater condition on ri $C$.

Then qualification (2) holds if int $(\operatorname{dom} f) \cap A \neq \emptyset$.

In fact, under the assumptions (a) and (b), Li et al. (2013) established the following FM-qualification for the system $\left\{f_{t}: t \in T\right\}$ :

$$
\text { epi } \delta_{C}^{*}+\sum_{i \in I} \text { cone }\left(\text { epi } f_{i}^{*}\right) \text { is closed. }
$$

Then one can apply again (Fang et al. 2010, Lemma 2.1(ii)) to verify that qualification (2) holds.

Acknowledgments The first author was supported in part by the National Natural Science Foundation of China (grants 11171300,11371325), and the second author was supported by Earmarked Grants (GRF) from the Research Grant Council of Hong Kong (Project nos. CUHK 403110 and 402612).

\section{References}

Dinh N, Goberna M-A, Lopez M-A, Mo T-H (2013) From Farkas to Hahn-Banach theorem. Preprint

Fang D-H, Li C, Ng K-F (2010) Constraint qualifications for extended Farkas's lemmas and Lagrangian dualities in convex infinite programming. SIAM J Optim 20(3):1311-1332

Farkas J (1902) Theorie der einfachen Ungleichungen. Journal für die Reine und Angewandte Mathematik $124: 1-27$

Goberna M-A, López M-A (1998) Linear semi-infinite optimization. In: Wiley series in mathematical methods in practice. Wiley, Chichester, UK

Jeyakumar V (2008) Farkas lemma: generalizations. Encyclopedia of optimization. Kluwer Academic Publishesr, Netherland, pp 87-91

Jeyakumar V, Dinh N, Lee G-M (2004) A new closed cone constraint qualification for convex optimization. In: Applied mathematics research report AMR04/8. School of Mathematics, University of New South Wales, Sydney, Australia

Li C, Zhao X-P, Hu Y-H (2013) Quasi-Slater and Farkas-Minkowski qualifications for semi-infinite programming with applications. SIAM J Optim 23(4):2208-2230 